\title{
Die neue Verfassung des Irak Aushandlungsprozess und institutionelles Design als Mittel zur Regelung der ethnischen Konflikte?
}

Mit dem Beginn des Verfassungsprozesses im Irak bot sich im Sommer 2005 dem Land die Möglichkeit, seine politischen Institutionen derart zu gestalten, dass diese den eigenen soziokulturellen und politischen Anforderungen gerecht werden. Mit der Ausarbeitung der neuen Verfassung erhielten die Akteure zugleich die Gelegenheit, einen allgemeinen Konsens über das zukünftige politische System im Irak herbeizuführen. Damit eröffnete sich die einzigartige historische Chance, die gewalttätigen Konflikte im Irak langfristig in politische Bahnen zu lenken. Eine solche institutionelle Konfliktregelung setzt jedoch einen transparenten, konsensorientierten Verfassungsprozess sowie ein den spezifischen Bedingungen angepasstes Verfassungsdesign voraus. Beide Anforderungen wurden bislang nicht erfüllt. Die Ablehnung der Verfassung durch die sunnitischen Araber und gravierende Mängel im institutionellen Design stellen dabei die zentralen Probleme dar. Dennoch fällt die Antwort auf die Frage, ob die historische Chance des verfassungsrechtlichen Neubeginns genutzt werden konnte, zwiespältig aus. Da der Verfassungsprozess noch nicht gänzlich abgeschlossen ist, erscheint eine institutionelle Konfliktregelung im Irak prinzipiell noch möglich - unter der Prämisse, dass der politische Prozess nicht einem völligen Abgleiten des Landes in einen offenen Bürgerkrieg zum Opfer fällt.

Inhalt

1. Verfassungsgebung als historische Chance zur gewaltfreien Konfliktregelung

2. Die irakische Geschichte als Einflussfaktor für die Zukunft des Landes? 827

3. Verfassungsgebung und Verfassungstechnik im Irak

a) Entstehung der Verfassung

b) Zentrale Verfassungsprinzipien

c) Institutionelle Regelsysteme

(1) Das Regierungssystem

(2) Das irakische Wahlsystem

(3) Die staatliche Organisationsstruktur

d) Potenzielle Auswirkungen auf den politischen Prozess 


\section{Verfassungsgebung als historische Chance zur gewaltfreien Konfliktregelung}

Für ein Land, das einen gewalttätigen Konflikt durchlaufen hat bzw. durchläuft, stellt die Aushandlung einer neuen Verfassung eine historische Chance dar. Der verfassungsrechtliche Neubeginn bietet die einzigartige Möglichkeit, politische Institutionen derart zu gestalten, dass sie den politischen Herausforderungen und soziokulturellen Eigenschaften dieses Landes gerecht werden. Die faktische institutionelle Ausgestaltung der Verfassung besitzt damit weitreichende Bedeutung. Dies gilt für die politische Entwicklung des Landes im Allgemeinen, im Besonderen aber für den Verlauf seines bislang gewalttätigen Konflikts. Damit entsteht die Möglichkeit, den Konflikt politisch zu regeln. ${ }^{1}$

Eine solche historische Chance erhielt der Irak im Sommer 2005. Zwei Jahre nach dem Ende der offiziellen Kampfhandlungen und der Etablierung eines Übergangsregimes begann man damit, eine neue Verfassung auszuarbeiten. Der Verfassungstext wurde im Oktober 2005 in einem landesweiten Referendum angenommen. Die sunnitischen Araber, die etwa $20 \%$ der irakischen Bevölkerung ausmachen, lehnten den Text allerdings mehrheitlich ab. Aufgrund dessen wurde zusätzlich vereinbart, weiterhin strittige verfassungsrechtliche Themen zu einem späteren Zeitpunkt durch Verfassungsänderungen zu klären.

Zwei Monate später fanden Parlamentswahlen statt. Die aus diesen Wahlen hervorgehende Regierung sollte als erste reguläre Regierung unter Anwendung der neuen Verfassung das Land lenken. Die Erwartungen richteten sich dabei primär auf die Beendigung der gewalttätigen Auseinandersetzungen. Dies sollte maßgeblich durch die Einbeziehung der sunnitischen Araber in den politischen Prozess erreicht werden. Die Regierungsbildung unter dem neuen Premierminister Nuri al-Maliki gestaltete sich jedoch äußerst schwierig. Sie wurde nicht nur spät, am 20.05.06, sondern auch unvollständig abgeschlossen, weil man sich nicht auf die Besetzung von Schlüsselressorts einigen konnte. Der angekündigte verfassungsrechtliche Konsens mit Vertretern der sunnitischen Araber steht weiterhin aus. ${ }^{2}$ Auch eine Stabilisierung der Sicherheitslage ließ sich seither nicht feststellen. Mit dem Amtsantritt der neuen Regierung stellt sich damit die Frage, ob die historische Chance, die sich dem Irak mit der Ausarbeitung einer neuen Verfassung im Hinblick auf eine gewaltfreie, demokratische und stabile politische Zukunft bot, genutzt werden konnte oder noch genutzt werden kann.

1 Die Grundannahme, dass Institutionen konfliktregulierend wirken können, geht maßgeblich auf Sir Karl R. Popper zurück, der diese Annahme seiner Theorie der Verfassungstechnik zugrunde legte (1974). Sie wurde von späteren Politikwissenschaftlern wie Giovanni Sartori weiterentwickelt und v. a. von Arend Lijphart und Donald Horowitz in jeweils unterschiedlicher Weise speziell auf fragmentierte Gesellschaften angewandt (Sartori 1997; Lijphart 1980, 1984; Horowitz 1985, 1990). Die Anwendung des institutionellen Konfliktregelungsansatzes im Irak erfolgt hier zudem auf der Grundlage und als Weiterentwicklung der eigenen Dissertation zu ethnisch-separatistischen Konflikten (Eichhorst 2005).

2 Anfang Oktober 2006 erfolgte allerdings ein erster Schritt auf diesem Weg, indem ein Verfassungsausschuss unter Einbeziehung aller irakischen Bevölkerungsgruppen mit dem Auftrag gebildet wurde, einen Konsens über Verfassungsänderungen zu erzielen. Dies zeigt, dass der politische Prozess trotz der zunehmenden Gewalt noch nicht gänzlich zum Erliegen gekommen ist. 
Irakische Verfassung

\section{Die irakische Geschichte als Einflussfaktor für die Zukunft des Landes?}

In der aktuellen Debatte über die Zukunft des Irak wird seine Vergangenheit häufig als Indikator eines Pro oder Kontra für die Chancen seiner demokratischen Entwicklung bemüht. Um die irakische Fähigkeit zur Demokratisierung unter Beweis zu stellen, wird u. a. auf demokratische Traditionen im frühen Mesopotamien verwiesen, deren Anfänge sich bereits unter den Sumerern im 3. Jahrtausend entwickelten (Davis 2005: 3 ff.). Weitere Beispiele, wie die jahrtausendalte Rechts- und Gesetzestradition des Landes als Basis aller Rechtsstaatlichkeit sowie erste Schritte zum Parlamentarismus während der britischen Kolonialzeit, ließen sich anführen (ausführlicher Tipp 2000).

Historische Parallelen werden jedoch auch gezogen, um Gegenteiliges zu konstatieren. So blickt der Irak auf eine lange Geschichte von Fremdherrschaft, autoritärer Systeme und politischer Instabilität zurück. Diese begann bereits in der Antike mit dem Aufstieg und Niedergang verschiedener Herrschaftsreiche (Babylonien, Assyrien und Neubabylonien) sowie diverser Herrschaftshäuser (Herrschaft Alexander des Großen, gefolgt von Parthern und Sassaniden). Von 630-640 n. Chr. folgte die Eroberung durch die Araber. Ab 930 geriet das Zweistromland unter wechselnde Fremdherrschaft von Persern und Mongolen. Im Jahr 1534 wurde der Irak schließlich von den Osmanen erobert und nach kurzzeitiger iranischer Herrschaft 1638 endgültig in das Osmanische Reich eingegliedert. Als Konstantinopel Ende des 19. Jahrhunderts zum „kranken Mann am Bosporus“ wurde, nutzte Großbritannien diese Schwäche, um seinen Einflussbereich auszudehnen. Zum Ende des Ersten Weltkriegs hatte das britische Königreich die faktische Kontrolle über den heutigen Irak etabliert. 1920 erhielt es für diesen ein Völkerbundmandat. Trotz der Erlangung der formalen Unabhängigkeit im Jahr 1932 blieb der britische Einfluss (besonders intensiv während der erneuten Besatzung im Zweiten Weltkrieg) bis Ende der 50erJahre bestehen.

Eine innenpolitische Stabilisierung oder gar Demokratisierung brachte die faktische staatliche Eigenständigkeit seit 1960 jedoch nicht. Das Land sah sich mit verschiedenen Minderheitenkonflikten konfrontiert, die noch aus der Zeit der Fremdherrschaft herrührten. Ebenso setzten sich innenpolitische Machtkämpfe zwischen britischen Loyalisten, Kommunisten, verschiedenen Fraktionen des Militärs und unterschiedlichen Flügeln der Baath-Partei fort. Erfolgreiche und weniger erfolgreiche Putschversuche waren die Folge und wurden in der Regel von der Proklamation provisorischer Verfassungen begleitet. Diese Entwicklung versetzte das Land mehrmals in bürgerkriegsähnliche Zustände. Seit Ende der 60er-Jahre begann schließlich Saddam Hussein unter der Präsidentschaft von Ahmad Hasan al-Bakr, seinen Einfluss auszudehnen. Mit seinem offiziellen Amtsantritt 1979 war dieser Prozess bereits abgeschlossen. Durch die Besetzung zentraler Ämter in Personalunion im irakischen Staatsapparat und innerhalb der Baath-Partei hatte er seine Machtposition als Staatspräsident konsolidieren können. Mit der Errichtung der Diktatur trat eine jahrzehntelange Phase des politischen Stillstands ein. Sie wurde erst mit dem Sturz Saddam Husseins im März 2003 beendet. Damit gelten die langjährigen Erfahrungen der Diktatur und Fremdherrschaft, die unstete und von Brüchen gekennzeichnete Ver- 
fassungsentwicklung sowie die fragmentierte Bevölkerungsstruktur häufig als unüberwindbare Hindernisse auf dem Weg eines demokratischen Transformationsprozesses.

Die Frage, ob sich der Irak demokratisch entwickeln wird, lässt sich allerdings nicht durch einen Blick auf seine Vergangenheit und die Unterstellung historischer GesetzmäBigkeiten beantworten (Popper 1974). Vielmehr müssen hierfür strukturelle Einflussfaktoren unter Berücksichtigung der Konfliktstruktur (Lipset/Rokkan 1967) des Irak analysiert werden. Seine Verfassung, mit der gleich ein ganzes Paket an strukturellen Einflussfaktoren geschnürt wurde, stellt dabei das zentrale Analyseobjekt dar. Die in ihr neu konzipierten Institutionen werden das Handeln der Menschen im Allgemeinen und ihr politisches Handeln im Besonderen langfristig steuern - unter der Voraussetzung, dass der politische Prozess nicht vollständig zum Erliegen kommt. Die neue Verfassung wird damit maßgeblichen Einfluss auf die weitere politische Entwicklung nehmen und sich speziell auf die Gruppenkonflikte auswirken. Dieser Wirkungszusammenhang erklärt die weitreichende Bedeutung, die dem Prozess der Verfassungsgebung im Irak zukommt.

\section{Verfassungsgebung und Verfassungstechnik im Irak}

Die allgemeine Annahme, dass Institutionen menschliches Handeln im Allgemeinen und politisches Handeln im Besonderen beeinflussen, stellt die Grundlage der klassischen Verfassungstechnik dar. ${ }^{3}$ Ihr Ziel im Kontext konfliktgeladener Gesellschaften ist es, ein für diese Gesellschaften passendes institutionelles Design zu entwickeln (Lijphart 1980, 1984; Horowitz 1985, 1990). Dieses soll den verschiedenen Bevölkerungsgruppen eines Landes die Möglichkeit geben, ihre Interessen auf politischem Wege durchzusetzen. Indem alle relevanten Akteure in den politischen Prozess integriert werden, soll die Möglichkeit entstehen, die bislang gewalttätigen Konflikte gewaltfrei zu regeln. Je fragmentierter die Gesellschaft eines Landes jedoch ist, je mehr Gruppen und infolgedessen je mehr Positionen miteinander konkurrieren, desto komplizierter gestaltet sich das Design der Institutionen.

Unter den Stichworten „Konflikttransformation“ und „Konfliktmanagement“ wurde der Verfassungstechnik in den vergangenen Jahren ein zweiter wissenschaftlicher Ansatz hinzugefügt. Während die Verfassungstechnik tatsächlich klassisches Verfassungsdesign und damit Themen wie die Gestaltung von Wahl- und Regierungssystemen umfasst, konzentriert sich der zweite Ansatz auf informelle Instrumente der gewaltfreien Konfliktregelung. Dazu zählen die Entwicklung vertrauensbildender Maßnahmen, Frühwarn-, Überwachungs- und Schlichtungssysteme durch neutrale Parteien oder auch GrassrootsInitiativen für ein gruppenübergreifendes, gewaltfreies Miteinander (ausführlicher Austin/ Fischer/Ropers 2004). Während das klassische Verfassungsdesign auf eine langfristige Wirkung angelegt ist, kann mit den beschriebenen informellen Instrumenten die unmittelbare Deeskalation einer akuten Konfliktsituation erreicht werden. Da die Anwendung der 
Verfassungstechnik ein hohes Maß an Kooperation und Konsens zwischen allen beteiligten Akteuren erfordert, ist ihre Implementierung in einer akuten Konfliktsituation nur schwer möglich. Daher können die informellen Instrumente dazu dienen, überhaupt erst die Voraussetzungen für die Anwendung der Verfassungstechnik zu schaffen. In diesem Sinne greifen beide Ansätze ineinander und ergänzen sich gegenseitig.

Die Notwendigkeit eines solchen Zusammenspiels ließ sich auch im Irak erkennen. Die dort angewandte Verfassungstechnik betraf nicht nur kleine Korrekturen an einzelnen institutionellen Regelungssystemen. Vielmehr wurden die institutionellen Grundlagen neu gestaltet. Die Funktionsweisen und Spielregeln des politischen Prozesses wurden gänzlich neu definiert. Dies wird sich langfristig auf die Möglichkeiten der Akteure, eigene Interessen innerhalb des politischen Prozesses effektiv durchzusetzen, direkt auswirken. Ein breiter gesellschaftlicher Konsens über die neue Verfassung war daher unerlässlich.

Die fragmentierte Bevölkerungsstruktur des Irak machte ihn umso dringlicher. Sie zeichnet sich durch zahlreiche Konfliktlinien aus, die die Bevölkerung entlang ethnischer, sprachlicher und religiöser Trennlinien in verschiedene Segmente spalten. Die Wahrnehmung und z. T. Instrumentalisierung dieser Trennlinien führte zu ethnischen Konflikten, d. h. zu Gegensätzen zwischen den Bevölkerungsgruppen, die sich aufgrund sprachlicher, religiöser, kultureller oder ähnlicher Faktoren als ethnisch distinkte Gruppen begreifen. Diese Gruppen, v. a. Schiiten, arabische Sunniten und Kurden (Letztere sind ebenfalls mehrheitlich sunnitisch), konkurrieren derzeit - insbesondere mit Gewalt - um die Durchsetzung ihrer Interessen. Um diese gewaltsamen Konflikte politisch zu regeln, erhält die Frage der politischen Einflussmöglichkeiten für alle Bevölkerungsgruppen ein besonderes Gewicht. Ein Konsens über die zukünftige politische Form des Landes, die über diese Durchsetzungsmöglichkeiten im politischen Prozess maßgeblich entscheidet, wurde damit zwingend notwendig.

Damit umfasste der Transformationsprozess zwei zentrale Aspekte: zum einen den politischen Prozess der Konsensfindung, der der Verfassung eine „formale Legitimation“ (Merkel 1999: 145) verleihen sollte; zum anderen die faktische institutionelle Ausgestaltung der neuen verfassungsrechtlichen Ordnung, mit der die „empirische Legitimation“ (Merkel 1999: 145) der Verfassung angestrebt wurde. In dem Sinne war es erforderlich, beide Ansätze zur gewaltfreien Konfliktregelung anzuwenden - den informellen während des Aushandlungsprozesses und den institutionellen für die inhaltliche Gestaltung des Verfassungstextes.

Ebenso wie beide Ansätze zur Konfliktregelung ineinander greifen, standen auch beide Aspekte des irakischen Transformationsprozesses in einem interdependenten Wirkungszusammenhang. Sollte das neue Regelwerk keine Akzeptanz innerhalb der Bevölkerung finden, wäre auch das kunstvollste institutionelle Design wirkungslos. In einem solchen Fall könnte sich in den betroffenen Bevölkerungskreisen die prinzipielle Ablehnungshaltung gegenüber der Verfassung festigen. Dagegen könnte ein alle Seiten überzeugendes und transparent entwickeltes institutionelles Design positiv auf die Akzeptanz der Verfassung und des Transformationsprozesses an sich hinwirken. Diese Interdependenz zwischen Verfassungsprozess und Verfassungsdesign erklärt die zentrale Bedeutung, die nicht nur der endgültigen Ausgestaltung, sondern auch bereits dem Entstehungsprozess der Verfassung zukommt. 
Um im Rahmen des Verfassungsprozesses das übergeordnete Ziel der Konsensbildung zu erreichen, sollten einige Anforderungen erfüllt werden. Dazu zählt, dass in dem Forum, in dem über die Verfassung verhandelt wird (im Irak das Verfassungskomitee), alle relevanten Gruppen repräsentiert sein sollten. Zudem sollte keine der Gruppen die Verhandlungen deutlich dominieren. Auch benötigt ein Aushandlungsprozess viel Zeit und Geduld, um durch Überzeugungsarbeit und ohne Gesichtsverlust für eine der Parteien eine Annäherung der verschiedenen Positionen erreichen zu können. Zeitdruck stellt daher eine prinzipiell ungünstige Voraussetzung dar.

Um die Legitimität und den Rückhalt der Verfassung in der Bevölkerung zu stärken, sollte diese ebenfalls in den Prozess einbezogen werden. Dieser erfordert daher ein hohes $\mathrm{Ma} ß$ an Transparenz. In öffentlichen Informationskampagnen sollten nicht nur die Inhalte, sondern auch das Zustandekommen bestimmter Entscheidungen vermittelt werden. Darüber hinaus sollte der Informationsfluss nicht einseitig verlaufen. Akteure aus allen Bereichen der Gesellschaft sollten die Möglichkeit erhalten, Vorschläge einzureichen und inhaltliche Anstöße zur Verfassungsgestaltung zu geben (Hart 2003; Benomar 2004).

Zu Beginn des Aushandlungsprozesses zeigte sich, dass man sich dieser Problematik bewusst war. Neben der US-amerikanischen und der britischen Regierung engagierten sich auch verschiedene Forschungsinstitute und Nichtregierungsorganisationen (NGOs) in diesem Prozess. Hierzu zählten Beratergruppen des National Democratic Institute (NDI), des United States Institute of Peace (USIP), des International Republican Institute (IRI) und der International Crisis Group (ICG). Auch staatliche Entwicklungshilfeorganisationen wie USAID assistierten hierbei. In der Phase zwischen Frühjahr und Herbst 2005 organisierte man zahllose Konferenzen zu Verfassungsfragen, bot Training, Beratung und technische Unterstützung für irakische NGOs an und unterstützte öffentliche Informationskampagnen.

Die zentrale Rolle im Verfassungsprozess kam allerdings den Vereinten Nationen zu. Mit der Resolution des Sicherheitsrats 1546 erhielten sie im Juni 2004 das offizielle Mandat, Unterstützung im politischen Prozess zu leisten. Die „UN Assistance Mission for Iraq“ (UNAMI) und hier insbesondere ihr „Office for Constitutional Support“ (OCS) begleiteten seitdem den politischen Prozess in allen Phasen. Zwischen Mai und Oktober 2005 leistete man Unterstützung, indem man einen nationalen Dialog über Verfassungsthemen beispielsweise durch öffentliche Diskussionsforen förderte. Expertenwissen wurde bereitgestellt und Medien- sowie Öffentlichkeitskampagnen wurden unterstützt. Nicht zuletzt ermöglichten die UN auch den Druck von mehr als drei Millionen Exemplaren des Verfassungsentwurfs und verteilten ihn an die Bevölkerung.

Die Verteilung des Verfassungsentwurfs bot jedoch auch ein Beispiel für die Probleme im Entstehungsprozess der Verfassung. Massive inhaltliche Konflikte zwischen den Verhandlungspartnern führten dazu, dass zeitweilig unterschiedliche Versionen des Verfassungstextes in Umlauf gebracht wurden. Nach Verlesung der ersten „endgültigen“ Version im Übergangsparlament am 28. August 2005 wurde am 13. September eine weitere ,end- 
gültige“ Version präsentiert, der am 18. September eine „,endgültige endgültige“ Fassung folgte. Diese wurde noch am selben Tag den Vereinten Nationen zum Druck übergeben. ${ }^{4}$ Die Verhandlungen mit sunnitischen Vertretern dauerten allerdings noch bis kurz vor Beginn des Referendums am 15. Oktober an. Veränderungen, die nach dem 18. September vereinbart wurden, waren deshalb nicht in der von den UN verteilten Version enthalten. So stimmte die Bevölkerung letztlich über eine Verfassung ab, deren genauer Inhalt ihr nicht bekannt war.

Auch scheiterte man bei dem Versuch, Anregungen aus der Bevölkerung aufzunehmen: Die Verfassungskommission hatte zwar Anfang Juni eine „Outreach Unit“ gegründet, die Fragebögen konzipierte und verteilte, auf denen Forderungen, Wünsche und Präferenzen der Befragten vermerkt werden konnten. Die Arbeit dieser „Outreach Unit“ erwies sich letztlich aber aufgrund von Zeitmangel als ineffektiv. Zwischen ihrer Gründung und dem Abgabetermin ihres Empfehlungsberichts lagen nur acht Wochen. Die Auswertung der ca. 150.000 ausgefüllten Fragebögen musste daher unter großer Eile vorgenommen werden. Letztlich wurde der Bericht zu spät an die Verfassungskommission übergeben, um seinen Inhalt noch berücksichtigen zu können. Damit erwies sich der Zeitfaktor als gravierendes Hindernis bei dem Versuch, die Bevölkerung in den Verfassungsprozess einzubeziehen (Morrow 2005).

Vergleichbares ließ sich in den Verhandlungen zwischen den politischen Akteuren beobachten. Nach den Wahlen vom Januar 2005, die von arabischen Sunniten mehrheitlich boykottiert worden waren, setzte sich das Übergangsparlament maßgeblich aus schiitischen und kurdischen Vertretern zusammen. Diese konnten sich erst am 28. April auf eine Übergangsregierung einigen. Eine Woche später wurde das 55-köpfige Verfassungskomitee ernannt. In diesem waren aufgrund der parlamentarischen Kräfteverhältnisse nur zwei Repräsentanten der sunnitischen Araber vertreten. Die Entscheidung über den Vorsitz für das Komitee fiel erst am 24. Mai. Da die Übergangsverfassung den 15. August als Abgabetermin für den Verfassungstext festlegte und die Möglichkeit der Fristverlängerung aus politischen Gründen nicht genutzt werden sollte, standen dem Komitee von Beginn an keine drei Monate zur Ausarbeitung der Verfassung zur Verfügung. In den ersten Wochen waren die sunnitischen Vertreter zudem unterrepräsentiert. Erst Mitte Juni wurden auf Druck der US-amerikanischen Regierung und des religiösen Schiitenführers Großayatollah Sayyid Ali al-Sistani 15 stimmberechtigte und zehn beratende sunnitische Vertreter in das Komitee aufgenommen (Brewer/Moller 2006).

Obwohl also die beteiligten Akteure die Notwendigkeit eines allgemeinen Verfassungskonsenses verstanden zu haben schienen, blieb kaum Zeit für eine Einigung. Sie kam letztlich auch nicht zustande. Insbesondere blieben die Positionen der Kurden und

4 Die juristische Korrektheit des gesamten Prozesses wurden anschließend bezweifelt. Dies betraf nicht nur das Fehlen einer parlamentarischen Abstimmung, sondern auch den Prozess der Fristenverlängerung, der nicht dem in der Übergangsverfassung vorgeschriebenen Prozedere entsprach. Wenngleich jedoch einige Bestimmungen nicht eingehalten wurden, war dennoch unzweifelhaft, dass die erforderliche Parlamentsmehrheit den Verfassungstext unterstützte. In diesem Sinne blieb das Ergebnis unverändert (International Crisis Group 2005). 
Schiiten auf der einen und der Sunniten auf der anderen Seite unvereinbar. Am 8. August wurden die Beratungen aus dem offiziellen Komitee in informelle Verhandlungsrunden zwischen schiitischen und kurdischen Politikern verlegt. Offiziell als schiitisch-kurdischer Führungsrat bezeichnet, wurden diese Runden auch als ,the kitchen“ bekannt (Morrow 2005). Die Sunniten nahmen an den Treffen nicht mehr teil. Ende August präsentierten Kurden und Schiiten einen fertigen Verfassungsentwurf. Wie vorauszusehen war, verweigerten die sunnitischen Vertreter ihm ihre Zustimmung.

Das Ziel eines transparenten, die Bevölkerung einbeziehenden und konsensorientierten Verfassungsprozesses war damit nicht erreicht worden. Um einen Konsens bemühte man sich zwar weiterhin, jedoch mit nur mäßigem Erfolg. Ein politisches Manöver der Kurden und Schiiten Anfang Oktober verschlechterte die Erfolgschancen zusätzlich: Beide Gruppen einigten sich auf einen veränderten Abstimmungsmodus für das Referendum, das den Sunniten de facto die Ausübung ihres Vetorechts unmöglich gemacht hätte. Auf amerikanischen Druck hin wurde diese Änderung wenige Tage später zurückgenommen. Dennoch wirkten die Maßnahmen der Kurden und Schiiten kontraproduktiv bei dem Versuch, ein Klima von Vertrauen und Kooperation zu schaffen und eine positive Einstellung der Sunniten gegenüber der neuen Verfassung zu erzeugen. Daran konnte auch die Zusage, im Anschluss an die Regierungsbildung erneut über Verfassungsänderungen zu verhandeln, nichts ändern. Bis auf eine sunnitische Partei, die Iraqi Islamic Party, empfahlen alle sunnitischen Parteien ihren Anhängern, den Verfassungsentwurf im Referendum abzulehnen.

Das Ergebnis des Referendums am 15. Oktober 2005 spiegelte die Konflikte bei der Ausarbeitung des Textes wider. Während eine breite Mehrheit der Gesamtbevölkerung für den Entwurf stimmte, wurde der Text in den primär sunnitisch besiedelten Gebieten deutlich abgelehnt. Die für ein Veto erforderliche Zweidrittelmehrheit in mindestens drei irakischen Provinzen wurde dennoch verfehlt. Die Verfassung gilt damit als angenommen.

Der Entstehungsprozess der Verfassung wurde somit den definierten Anforderungen nicht gerecht. Ein allgemeines Bewusstsein um die Schwierigkeiten eines solchen Unterfangens war zwar auf allen Seiten zu erkennen. Dennoch wurde kein Konsens erreicht. Die Unfähigkeit, einen gemeinsamen Text zu formulieren, lässt sich primär auf zwei Gründe zurückführen: zum einen auf die tief greifenden Meinungsverschiedenheiten zwischen den beteiligten Akteuren ${ }^{5}$, zum anderen auf die Verhandlungsstrategie der US-Regierung.

Sie wirkte zwar in weiten Teilen positiv auf den Verfassungsprozess ein, indem sie alle Akteure zur Kooperation anhielt, die Einbeziehung der Sunniten durchsetzte, auf der Beibehaltung des Abstimmungssystems im Referendum bestand und letztlich die Zusage zu weiteren Verhandlungen erwirkte. Gleichzeitig aber hielt sie konsequent an einer selbst gesetzten Zeitmarke fest, deren Verschiebung die Übergangsverfassung ohne Weiteres zugelassen hätte. Damit wurde künstlich ein immenser Zeitdruck geschaffen, der sich kon-

5 Sie bestanden v. a. hinsichtlich der Gestaltung der staatlichen Organisationsstruktur, der Rolle der Religion, der Verteilung der Öl- und Gasressourcen, der umstrittenen „Ölstadt“ Kirkuk sowie der Rolle der Milizen. 
traproduktiv auf alle Aspekte des Verfassungsprozesses auswirkte. Der Zeitdruck verhinderte eine effektive und umfassende öffentliche Informationskampagne sowie inhaltliche Diskussionen und den Austausch mit der Bevölkerung. Letztlich führte er auch zum Ausschluss der Sunniten aus den Verhandlungen. Damit wurde der Einhaltung der Fristen auf Kosten der Konsensbildung Priorität eingeräumt. Mit Blick auf das institutionelle Design der Verfassung drängt sich zudem der Eindruck auf, dass unter dem zeitlichen Druck nicht nur der Entstehungsprozess der Verfassung, sondern auch die Qualität des Verfassungsdesigns gelitten hat.

\section{b) Zentrale Verfassungsprinzipien}

Eine wesentliche Anforderung an die inhaltliche Gestaltung der Verfassung eines Landes mit konfliktgeladener Bevölkerungsstruktur ist die Einigung auf fundamentale Prinzipien und die Anerkennung internationaler Rechtsstandards. Dazu zählen in erster Linie die allgemeinen Menschenrechte, die Verpflichtung zur Anerkennung demokratischer Werte sowie Minderheitenrechte.

Die irakische Verfassung erfüllt diese Anforderung im Großen und Ganzen. ${ }^{6}$ In Art. 1 schreibt sie den demokratischen Charakter des Landes fest und erkennt in Art. 3 seinen multiethnischen Charakter an. Auch sind in ihr universelle Menschenrechte verankert: Kapitel 2 definiert zunächst zivile und politische Rechte (Art. 14-34) und zählt anschließend persönliche Freiheitsrechte auf (Art. 35-45). Alle ethnischen Gruppen werden ausdrücklich gleichberechtigt nebeneinander gestellt. Da der sprachlichen Konfliktlinie in ethnischen Konflikten häufig eine entscheidende Rolle zukommt, ist die Verankerung von weitgehenden sprachlichen Rechten in Art. 4 bedeutend. ${ }^{7}$ Diese gelten v. a. den Kurden, aber auch kleineren Minderheiten, wie Turkmenen und Chaldo-Assyrern, werden erstmals sprachliche Rechte zugesprochen. Artikel 135 garantiert zudem allen Minderheiten politische, kulturelle, administrative und bildungspolitische Rechte.

Trotz der insgesamt positiven Bilanz sind aber auch einige Einschränkungen zu verzeichnen. So kennt die Verfassung keine Ewigkeitsklausel in Bezug auf ihre grundlegenden Werte. Eine Änderung des ersten Kapitels würde sich nach Art. 136 Abs. 2 zwar kompliziert gestalten, wäre aber prinzipiell möglich. Auch wird die Verankerung der Men-

6 Im Folgenden wird nur auf Verfassungsartikel eingegangen, in denen de facto zentrale Verfassungsprinzipien festgeschrieben werden und die daher für die hier behandelte Thematik relevant sind. Gleiches gilt für den Abschnitt über institutionelle Regelsysteme, in dem auch nur die tatsächlich thematisch relevanten Verfassungsartikel berücksichtigt werden.

7 Die Muttersprache als primäres Identifikationsmerkmal ist unveränderlich und trägt damit kontinuierlich zur Perzeption der Andersartigkeit zwischen unterschiedlichen Sprachgruppen bei. Zudem kann sie sich intensivierend auf weitere Konfliktlinien auswirken. Stellt etwa die Muttersprache ein Hindernis bei der Schulbildung dar, kann sie zusätzlich zur Entstehung oder Vertiefung sozioökonomischer Konfliktlinien beitragen. Dies war beispielsweise in Quebec in den 50er- und 60er-Jahren der Fall, als frankophone Kanadier auf Grund von Sprachbarrieren ein deutlich geringeres Einkommen als anglophone Kanadier aufwiesen (Eichhorst 2005: 67 ff.). 
schenrechte durch Art. 44 relativiert. Dieser ordnet internationale Menschenrechtskonventionen den Prinzipien und Regeln der Verfassung unter. Der Bezug auf den Islam als eine grundlegende Rechtsquelle (Art. 2 und Art. 2 a bei gleichzeitiger Bindung an demokratische Werte (Art. 1 und Art. 2 b) mag manchem als Paradoxon erscheinen, obgleich hier von ,den unstrittigen Regeln des Islams“ und nicht von ,islamischem Recht“ gesprochen wird. Artikel 36 wiederum garantiert die Meinungs-, Presse- und Versammlungsfreiheit nur dann, wenn dadurch die öffentliche Ordnung oder Moral nicht beschädigt oder angegriffen wird. Da es sich dabei um dehnbare Begriffe handelt, könnten sie häufiger als notwendig bemüht und Freiheitsrechte willkürlich eingeschränkt werden. In welche Richtung sich die Rechtsprechung und Verfassungsinterpretation dieser zahlreichen ambivalenten Formulierungen entwickeln werden, muss daher zunächst abgewartet werden.

\section{c) Institutionelle Regelsysteme}

Neben fundamentalen Prinzipien und universellen Grundsätzen enthält eine Verfassung gleich ein ganzes Set an institutionellen Regelsystemen. Von diesen lassen sich drei nennen, die den politischen Prozess am nachhaltigsten beeinflussen: das Regierungssystem, das Wahlsystem und die staatliche Organisationsstruktur.

\section{(1) Das Regierungssystem}

Unter der bisherigen Übergangsverfassung ließ sich das irakische Regierungssystem noch als semipräsidentielles System bezeichnen. In diesem kam sowohl dem dreiköpfigen Präsidialrat als auch dem Premierminister eine zentrale politische Funktion zu (Eichhorst/ Sinjen 2005b). Mit dem Inkrafttreten der endgültigen Verfassung ${ }^{8}$ und nach Ende der Übergangsphase $^{9}$ kann das irakische Regierungssystem - unter Berücksichtigung des Wahlsystems - als quasi-parlamentarisches System ${ }^{10}$ bezeichnet werden: Der Präsidialrat wird durch einen regulären Präsidenten (Art. 65-73) ersetzt, der als Staatsoberhaupt primär repräsentative Aufgaben wahrnimmt. Die Besetzung dieses Amtes erfolgt zu Beginn der Legislaturperiode und benötigt eine Zweidrittelmehrheit. Die Wahl des Präsidenten steht somit am Anfang jeder Regierungsbildung. Anschließend ernennt der Präsident den

8 Die folgende Darstellung bezieht sich auf den Verfassungstext, der auf der Website der irakischen Übergangsregierung veröffentlicht ist (http://www.iraqigovernment.org/constitution_en.htm).

9 Die Verfassung definiert die aktuelle Legislaturperiode als Übergangsphase, in der einige Bestimmungen der Übergangsverfassung weiterhin gelten. Die Legislaturperiode umfasst vier Jahre.

10 Als quasi-parlamentarisches System wird hier in Abgrenzung zum klassischen Westminster-Modell mit seinem Mehrheitswahlsystem ein parlamentarisches System mit Verhältniswahlrecht bezeichnet. Diese begriffliche Unterscheidung wird häufig unterlassen, doch erscheint sie aufgrund der diversen Ausprägungen parlamentarischer Systeme angebracht. Giovanni Sartori konstatiert in diesem Zusammenhang: „Parliamentary systems differ so widely among themselves as to render their common name a misnomer for a deceitful togetherness" (1997: 83). 
Premierminister. Dieser steht an der Spitze der Regierung (Art. 74-84) und ist vom Vertrauen des Parlaments (Art. 47-62) abhängig. Verliert der Premierminister dieses, muss das gesamte Kabinett zurücktreten. Der Premierminister nimmt damit die Stellung des Primus inter Pares ein. Als solcher muss er mit einem Parlament zusammenarbeiten, das über umfangreiche Kompetenzen verfügt.

So kann es nicht nur dem Premierminister, sondern auch allen anderen Kabinettsmitgliedern mit absoluter Mehrheit das Vertrauen entziehen. Dem Parlament kommt damit ein großes Maß an Verantwortung für die Stabilität oder Instabilität der Regierung zu, die nicht nur aktuell, sondern vermutlich auch in Zukunft aus sorgsam ausgehandelten Koalitionen bestehen wird. Gleichzeitig verfügt der Premierminister über kein eigenständiges Auflösungsrecht, sondern benötigt dafür die Zustimmung des Parlaments und des Präsidenten. Das Parlament kann sich dagegen mit absoluter Mehrheit auch selbst auflösen.

Ein weiteres Beispiel ist die gesetzliche Regelung von mehr als 50 Themengebieten, die verfassungsrechtlich bislang nicht festgeschrieben wurden. Teilweise sind die betroffenen Themengebiete von weitreichender Bedeutung. Dazu zählt die Ausgestaltung der zweiten Parlamentskammer, des „Council of Union“ (Art. 63), die in der Verfassung eine nur rudimentäre Form erhält. Dem Parlament als erster Kammer ein solches Maß an Verfügungsbefugnis über die Errichtung der zweiten Kammer zu geben, gilt als ungewöhnlich (Brown 2005). Dem Parlament wird so eine verfassungsgestaltende Rolle zugeschrieben. Es kann als sicher angenommen werden, dass es seine dominante Rolle auch für die Zukunft festschreiben wird, indem es der zweiten Kammer nur geringe legislative Kompetenzen zuweist - sollte dies überhaupt geschehen. Eine gleichberechtigte Stellung beider Kammern wird damit unwahrscheinlich. Dies wird sich letztlich auch auf die Ausprägung des angestrebten föderalen Systems auswirken.

In erster Linie symbolischer Natur ist das Recht des Parlaments, den Staatspräsidenten vorzuladen. Da dieser als Staatsoberhaupt dem Parlament politisch nicht verantwortlich ist und nur bei schwerwiegendem Fehlverhalten abgesetzt werden kann, gilt dieses Interpellationsrecht dennoch als außergewöhnlich. Es unterstreicht erneut die zentrale Position, die dem Parlament im Verfassungsdesign zugeschrieben wurde.

Eine derart starke Legislative gilt als begünstigende Voraussetzung für einen erfolgreichen demokratischen Transformations- und Konsolidierungsprozess (Fish 2006). Über diesen Aspekt hinaus weist das Regierungssystem keine Merkmale auf, die darauf hindeuten, dass es speziell den irakischen Bedürfnissen angepasst wurde. Insbesondere fehlt es an langfristig gesicherten Mechanismen, die eine Integration ethnischer Minderheiten sichern könnten. Vielmehr entfallen mit der endgültigen Verfassung und dem Ende der Übergangsphase entsprechende Regelungen, die in der Übergangsverfassung noch enthalten waren.

Dies gilt insbesondere mit Blick auf das Präsidentenamt, das in der Übergangsverfassung noch als Präsidialrat und damit als Kollegialorgan ausgestaltet war und über politische Kompetenzen verfügte. Die bislang bestehende Möglichkeit, Kurden, Sunniten und Schiiten gleichermaßen in einem Organ repräsentiert zu sehen, entfällt damit. Aufgrund der erforderlichen Zweidrittelmehrheit für die Ernennung des Präsidenten werden Aus- 
handlungsprozesse, die zugleich die Besetzung des Premierministeramtes einschließen, vermutlich weiterhin stattfinden, doch entfällt deren Institutionalisierung.

Aber auch die Aushandlung ist nicht mehr zwingend notwendig: Wird keine Einigung auf einen Präsidentschaftskandidaten erreicht, erfolgt eine Stichwahl zwischen den beiden Kandidaten mit den meisten Stimmen. Eine absolute Mehrheit wäre damit für den Beginn der Regierungsbildung bereits ausreichend. Bei der aktuellen Stimmenverteilung im Parlament bedeutet dies, dass sich in einer solchen Situation theoretisch nur noch zwei Gruppierungen - beispielsweise Schiiten und Kurden unter Ausschluss der Sunniten - auf einen Kandidaten einigen müssten; bei einer absoluten Mehrheit der schiitischen Allianz nur noch eine. Gleiches gilt für das anschließende politische Tagesgeschäft. Sollte sich also in der politischen Praxis keine gegenteilige Übung etablieren, wie die traditionelle Bildung einer „Regierung der nationalen Einheit“, wird mit dem Regierungssystem der neuen Verfassung ein - mehr oder minder - reines Konkurrenzmodell institutionalisiert.

Die Verfassung schreibt im Art. 48 kein spezifisches Wahlsystem vor, sondern überlässt dies der Ausgestaltung durch das Parlament. Lediglich einige allgemeine Anforderungen werden vorgegeben. Dazu zählt die Absicht, im Parlament eine Repräsentation aller Gruppen der Gesellschaft zu erzielen. Dies kann als Festschreibung eines Proporzsystems verstanden werden, ist jedoch nicht zwingend notwendig. Zudem findet sich unter Art. 151 die aus der Übergangsverfassung stammende Richtlinie wieder, die einen Frauenanteil von $25 \%$ unter den Abgeordneten vorgibt. Da sie aber im abschließenden Kapitel „Übergangsbestimmungen“" steht, wird sie wahrscheinlich nach Ende der Übergangsfrist entfallen.

Der Blick muss deshalb auf die einfache Gesetzgebung gelenkt werden. Der Irak hat damit in der bislang kurzen Geschichte seiner im Großen und Ganzen als frei und fair beurteilten Wahlen bereits einige Erfahrungen sammeln können. Seit dem Ende der Diktatur haben zwei Parlamentswahlen stattgefunden. Bei der ersten Wahl im Januar 2005 wurde ein reines Proporzsystem angewandt, bei dem das gesamte Staatsgebiet als ein einziger Wahlkreis behandelt wurde (Eichhorst/Sinjen 2005a). In den sunnitisch dominierten Gebieten wurden die Wahlen teils boykottiert, teils war eine Teilnahme aufgrund der instabilen Sicherheitslage nicht möglich. Als Folge davon war die sunnitische Bevölkerung im Parlament kaum repräsentiert.

Zeitliche und logistische Gründe hatten zur Entscheidung für dieses Wahlsystem geführt. Die Einteilung des Staatsgebietes in Wahlkreise hätte genaue Kenntnisse über die exakte Bevölkerungsverteilung vorausgesetzt. Auch die Parteien wären vor Schwierigkeiten gestellt worden, da sie Kandidaten für möglichst alle Wahlkreise hätten aufstellen müssen (ausführlicher Dawisha/Diamond 2006: 92 f.). Die Mängel dieses Wahlsystems sollten bis zu den Wahlen im Dezember 2005 behoben werden. Daher etablierte man nun erstmalig (Mehrmann-)Wahlkreise, die mit den 18 irakischen Provinzen identisch waren. Auf der Grundlage der Daten des UN-Nahrungsmittelprogramms wurden diesen Provin- 
zen entsprechend ihrem Bevölkerungsanteil Parlamentsmandate zugeteilt. Das aktuelle Wahlsystem sieht nun zudem einen nationalen Stimmenausgleich vor. So werden von den 275 Parlamentssitzen nur 230 als Wahlkreismandate nach einfachem Wahlzahlverfahren verteilt. Die übrigen 45 Mandate werden zunächst an diejenigen Parteien vergeben, die in den Wahlkreisen keine Mandate erhalten haben, aber die „nationale Wahlquote“ dennoch erfüllen. ${ }^{11}$ Die anschließend noch übrig bleibenden Sitze werden den Parteien in einem letzten Schritt entsprechend deren nationaler Stimmenanteile zugeteilt (Electoral Commission of Iraq 2006). Mit dieser Form des Wahlsystems wurde die Proporzanforderung, die einige Beobachter aus der Verfassung ableiteten, erfüllt.

\section{(3) Die staatliche Organisationsstruktur}

Der Irak erhält mit seiner neuen Verfassung ein stark dezentralisiertes Autonomiesystem. Die Liste der exklusiven Kompetenzen der Zentralebene gestaltet sich außergewöhnlich kurz (Art. 108 Abs. 1-9). Selbst klassische Zuständigkeiten wie die Außenpolitik werden eingeschränkt, indem den Subsystemen das Recht auf internationale Repräsentation in den diplomatischen Vertretungen des Irak zugestanden wird (Art. 116 Abs. 5.). Zugleich spricht Art. 111 alle nicht aufgezählten Kompetenzen grundsätzlich den Regionen zu. Deren zahlreiche Zuständigkeiten und Strukturen werden detailliert in den Artikeln 113-131 aufgeführt. Zudem wird den rechtlichen Vorgaben der Regionen im Bereich der geteilten Zuständigkeiten im Falle von Streitigkeiten Vorrang eingeräumt. Zu diesen geteilten $\mathrm{Zu}$ ständigkeiten zählt u. a. die Verwaltung und Verteilung der Öl- und Gasressourcen, die in den Artikeln 109 und 110 durchaus widersprüchlich und potenziell konfliktreich formuliert wurde. ${ }^{12}$ Auch sind die Regionalparlamente befugt, die Implementierung nationaler Gesetze im eigenen Sinne zu verändern, sollten Widersprüche zwischen nationalem und regionalem Recht entstehen und diese thematisch nicht in den Bereich der exklusiven Kompetenzen der Zentralebene fallen.

Von eminenter Bedeutung ist auch Art. 129. Dieser schreibt den Regionalregierungen die Zuständigkeit für die innere Sicherheit zu. Das Polizeirecht an sich stellt dabei keine ungewöhnliche Kompetenz dar. Der Begriff „,regional guards“ allerdings scheint lediglich ein anderes Wort für „militias“ zu sein. Damit konnten sich die Kurden das Existenzrecht ihrer „Peshmerga“-Milizen sichern. Wenngleich dieser Artikel bislang nur für tatsächlich bestehende Regionen Gültigkeit besitzt, werden sich aber auch sunnitische und schiitische Milizen in ihrer Existenz legitimiert sehen. Die aktuellen Vorwürfe, diese Milizen würden

11 Dieses Verfahren ist ebenfalls ein einfaches Wahlzahlverfahren. Die nationale Wahlquote wird ermittelt, indem die Gesamtzahl der abgegebenen Stimmen durch die Gesamtzahl der Mandate (275) dividiert wird. Diese Quote erreichte in den Dezemberwahlen nur eine Partei, die auf diesem Wege auch nur ein Mandat erzielen konnte.

12 Insbesondere Art. 110 Abs. 2 sorgte unter sunnitischen Arabern für Unmut, da dieser die besondere Berücksichtigung von Regionen, die zu Zeiten Saddam Husseins ,unfair“ behandelt wurden, vorsieht. 
Angriffe auf die jeweils andere Bevölkerungsgruppe durchführen, lässt das Konfliktpotenzial dieser Regelung erahnen.

Ein weiterer Aspekt des Autonomiemodells ist die Unterscheidung von Provinzen und Regionen. Letztere sind mit deutlich größeren Kompetenzen ausgestattet. Die bislang einzige Region ist die kurdische im Norden des Irak. Artikel 114 ermöglicht es jedoch allen Provinzen, eine Region zu bilden und damit die weitgehende Autonomie zu erlangen, die die Verfassung den Regionen zuspricht.

Das wesentliche Merkmal der irakischen Organisationsstruktur ist jedoch ihr mangelnder föderaler Charakter. Obwohl als „Föderalismus“ betitelt, handelt es sich um kein föderales System im eigentlichen Sinne. Es existiert derzeit noch keine föderale zweite Parlamentskammer, in der den Provinzen und Regionen Mitwirkungsrechte auf nationaler Ebene zugesichert würden. Wie oben bereits erläutert, ist zudem kaum zu erwarten, dass eine solche Kammer mit außergewöhnlich breiten Kompetenzen ausgestattet wird. Damit stärkt das aktuelle System v. a. die Eigenständigkeit der Regionen und entspricht insbesondere kurdischen Interessen. So wurde in der Verfassung ein Autonomiesystem festgeschrieben, das in seinen Grundzügen stark an das asymmetrische Autonomiemodell in Spanien erinnert (Eichhorst 2005: 185 ff.).

\section{d) Potenzielle Auswirkungen auf den politischen Prozess}

Die Entwicklung der irakischen Verfassung ist keineswegs optimal verlaufen.. Damit drohen negative Auswirkungen. Der fehlende Konsens lässt befürchten, dass die irakische Verfassung von den sunnitischen Bevölkerungskreisen dauerhaft abgelehnt wird. Die Erfahrungen in Kanada, Spanien und Sri Lanka belegen, dass sich dies langfristig auch negativ auf die Identifikation einzelner Bevölkerungsgruppen mit dem Staat und ihre Integration auswirken kann (Eichhorst 2005: 103 ff., 148 f., 233 f.). Insbesondere in Konfliktsituationen, in denen die Verfassung konfliktregulierend wirken sollte, wird ihre Legitimität angezweifelt und damit auch ihr Lösungsansatz abgelehnt. Nicht selten kommt es zur Instrumentalisierung dieser Sachlage: Die betroffenen Gruppen vertreten die Meinung, sie seien an verfassungsrechtliche Vorgaben, denen sie nie zugestimmt haben, auch nicht gebunden. Dies ist auch im Irak zu befürchten.

Untermauert wird diese Einschätzung durch die Analyse des Verfassungsdesigns. Insbesondere die Gestaltung des Regierungssystems lässt eine Berücksichtigung der spezifischen Bedingungen im Irak vermissen. Die Errichtung eines Mehrheitssystems ist in Ländern mit homogener Bevölkerungsstruktur üblich und entspricht der ursprünglichen Logik des politischen Wettbewerbs. In einer fragmentierten Gesellschaft wie der des Irak kann es jedoch verheerende Auswirkungen auf den weiteren politischen Prozess haben. Dort sind Mittel erforderlich, die die Beteiligung aller relevanten Akteure am politischen Prozess des Landes garantieren. Sehen sich die Minderheiten außerstande, ihre vitalen Interessen innerhalb des Systems durchzusetzen, werden sie langfristig andere Wege wählen. Im Falle der Kurden wäre dies möglicherweise die Sezession „Kurdistans“, im Falle der Sunniten die Fortführung des gewaltsamen Protests gegen den Transformationspro- 
zess. Obwohl in den vergangenen Jahren verschiedene Mechanismen zur Minderheitenintegration entwickelt wurden (ausführlicher Bächler 2004), wurden sie nicht in die irakische Verfassung eingebaut. Bis auf einen schwachen Konsenszwang zu Beginn der Regierungsbildung lassen sich keine institutionellen Garantien für Minderheiten erkennen. Insbesondere mangelt es an einem Minderheitenveto für deren vitale Interessen. Aber auch andere Möglichkeiten, wie beispielsweise die Festschreibung eines ethnischen Proporzes für das Kabinett, wurden nicht genutzt.

Die Bildung einer sogenannten Regierung der nationalen Einheit wie der aktuellen ist damit in Zukunft nicht gesichert. Schwinden die derzeitige Bereitschaft zum Konsens und der entsprechende internationale Druck, Minderheiten an der Regierungsbildung zu beteiligen, gibt es daher kaum noch Garantien für diese Gruppen, ihre Ziele durchzusetzen. Solange zudem ihr gewaltsamer Widerstand als einziges Argument für die Integration der Sunniten angeführt wird und keine institutionellen Garantien für ihre politische Beteiligung existieren, besteht für sie kein Anreiz, ihre Gewalt aufzugeben. Vielmehr sichern sie sich hierdurch ihr politisches Mitspracherecht und die Rücksichtnahme auf ihre Positionen und Empfindlichkeiten. Damit ist die Demonstration von Gewalt derzeit ihre einzige Möglichkeit, im politischen Prozess Gehör zu finden. Dies unterstreicht die Notwendigkeit, institutionelle Anreize zu schaffen, um alle Gruppen zu einer gewaltfreien Teilnahme am politischen Prozess zu bewegen.

Die Wirkungsweise des Wahlsystem ließ sich bei den Wahlen vom Dezember 2005 bereits beobachten. Es wurde den Erwartungen seiner Konstrukteure überwiegend gerecht: Nahezu alle Bevölkerungsgruppen sind aktuell im Parlament entsprechend ihrem ungefähren Anteil an der Gesamtbevölkerung vertreten. Dies gilt auch für die Sunniten, die an den Dezemberwahlen mit großer Mehrheit teilnahmen. Obgleich damit ein zentrales Ziel erreicht werden konnte, ist dennoch fraglich, ob das Wahlergebnis in jeder Hinsicht optimal ausgefallen ist. So ließ sich im Vergleich zu den Januarwahlen eine zunehmende Fragmentierung und Polarisierung des Parteiensystems feststellen (Eichhorst/Sinjen 2006: 10 ff.). Der Grund hierfür waren u. a. mangelnde Anreize im Wahlsystem für Zusammenschlüsse und für die Vertretung moderater Positionen. Das Proporzsystem ermöglichte es den Akteuren vielmehr, mit äußerst speziellen, zumeist ethnisch geprägten Agenden erfolgreich Wahlkampf zu betreiben.

Damit lässt sich im Bereich des „electoral engineering“ ein veritables Dilemma konstatieren: Sollen Minderheiten auf der einen Seite politisch repräsentiert werden, wäre es gleichzeitig für die Stabilität des politischen Prozesses dringend erforderlich, Fragmentierung und Polarisierung im Parteiensystem vorzubeugen (Sartori 1976). Entscheidende Bedeutung erlangt in dieser Hinsicht zudem ein grundsätzlicher Wissenschaftsstreit, der sich nicht nur auf die Frage des Wahlsystems beschränkt, darin aber offen zutage tritt. So herrscht über die Frage, wie ein ,perfektes“ Institutionengefüge und insbesondere ein „perfektes“ Wahlsystem auszusehen hat, in der wissenschaftlichen Debatte keine Einigkeit. Anhänger des Konkordanzansatzes nach Lijphart vertreten die Auffassung, die politischen Realitäten fragmentierter Gesellschaften müssten anerkannt und im Verfassungsdesign berücksichtigt werden. Kritiker dieses Ansatzes befürworten dagegen Techniken, die 
„ethnische Politik“ unterbinden und Anreize für moderates, gruppenübergreifendes Handeln kreieren, da ethnische Politik destabilisierend wirke (Horowitz 1990).

Im Bereich des ,electoral engineering“ haben sich in diesem Rahmen Ansätze herausgebildet, die die Entstehung ethnischer Parteien zu verhindern versuchen. Dies soll u. a. durch Vorgaben in Parteiengesetzen ermöglicht werden. Derartige Maßnahmen sind aber häufig äußerst restriktiv, indem sie Parteien z. B. verbieten, ethnische oder regionale Symbole zu verwenden. Auch wird Parteien mitunter vorgeschrieben, sich überregional zu organisieren, um „ethnische Politik“ zu verhindern (Reilly 2003: 10 f.). Es ist allerdings mehr als fraglich, ob dieser Ansatz im Irak politisch umgesetzt werden kann. Insbesondere die kurdischen, letztlich aber nahezu alle irakischen Parteien sind regional verankert und haben ethnische Grundlagen. Eine Zustimmung zu derartigen Anforderungen würde sie vor immense Herausforderungen stellen und letztlich ihre Machtbasis gefährden.

Ein zweiter Ansatz ist die Beeinflussung des Parteiensystems über das Design des Wahlsystems. In den vergangenen Jahren wurden zahlreiche Wahlsysteme entworfen, die zu moderatem politischen Handeln anhalten sollen (Reilly 2001). Abgesehen von Neuentwicklungen bietet sich aber auch das klassische relative Mehrheitswahlrecht an. Diesem eilt zwar der Ruf voraus, Minderheiten aufgrund seiner äußerst hohen, systemimmanenten Prozenthürde vom politischen Prozess auszuschließen. Allerdings trifft das nicht auf Länder zu, in denen die Minderheiten geografisch kompakt siedeln. Vielmehr ist das Gegenteil der Fall. Die Tendenz des relativen Mehrheitswahlsystems, regionale Hochburgen überdurchschnittlich zu honorieren, führt häufig zu einer überdurchschnittlichen Repräsentation dieser Gruppen (Eichhorst 2005: 94 ff., 269 ff.).

Im Falle von kompakt siedelnden Minderheiten kann also ein Mehrheitswahlsystem ihre besonders effektive Repräsentation gewährleisten und gegebenenfalls zu ihrer Einbeziehung in die Regierungsbildung beitragen. Gleichzeitig ist eine übermäßig Fragmentierung kaum zu erwarten, da das Mehrheitswahlsystem trotz allem zum politischen Zusammenschluss anhält. Auch etabliert es ein enges Verhältnis zwischen dem einzelnen Wahlkreisabgeordneten und seinem Wahlkreis. Auf diese Weise wird eine gewisse Unabhängigkeit der Kandidaten von ihrer Partei gewährleistet. Damit kann innerparteilicher Korruption, „Postengeschacher“ und Entfremdung von der Basis vergleichsweise effektiv vorgebeugt werden. Es kann so zur Optimierung des politischen Prozesses und zur Legitimation des Systems innerhalb der Bevölkerung beitragen. Ethnische Politik kann dieses Wahlsystem allerdings nicht verhindern.

Es ist jedoch fraglich, ob die Verhinderung ethnischer Politik im Irak derzeit das primäre Ziel sein sollte. Die ethnische Fragmentierung des Irak stellt eine politische Realität dar, die sich bereits vor Jahrzehnten in der Bildung ethnischer Parteien niedergeschlagen hat. Ihre jahrzehntelange Existenz widerlegt häufig wiederholte Behauptungen, die diversen Konfliktlinien des Landes seien erst mit Beginn des dritten Golfkrieges entstanden. Darüber hinaus genügt ein Blick auf die 20er- und 30er-Jahre sowie auf spätere Entwicklungen insbesondere im kurdischen Nordirak, um die lange „Tradition“ ethnischer Konflikte im Irak zu erfassen. Ethnische Politik wird damit bereits seit langer Zeit betrieben (Dawisha 2005: 48). Sie nun durch Verfassungstechnik unterbinden zu wollen, erscheint 
praktisch unmöglich und die Durchsetzung derartiger Techniken, wie beispielsweise eines Verbots ethnischer Parteien, höchst unwahrscheinlich.

Ethnische oder religiöse Identitäten als solche müssen zudem keineswegs per se negativ beurteilt werden. Insbesondere in Zeiten radikaler politischer Umbrüche können derartige Identitäten auch Stabilität und Sicherheit vermitteln. Negativ sind ihre Auswirkungen dann, wenn sie für politische Zwecke instrumentalisiert werden und zu gewalttätigen Zusammenstößen mit anderen Bevölkerungsgruppen führen. Dies zu verhindern, sollte das vorrangige Ziel der Verfassungstechnik im Irak sein. Wichtig wäre hierfür v. a. die wirklich föderale Gestaltung der staatlichen Organisationsstruktur.

Dies aber wurde im Irak nicht realisiert. Die irakische Verfassung garantiert zwar eine äußerst weitgehende Autonomie der Provinzen und Regionen, versäumt aber zugleich, diese über Mitwirkungsrechte auf nationaler Ebene effektiv einzubinden. Indem es die Autonomie fördert, die Integration in den Staat aber nicht forciert, verstärkt es die zentrifugalen Tendenzen, anstatt ihnen entgegenzuwirken. Damit gleicht das irakische System gegenwärtig stärker dem Autonomiesystem nach spanischem Vorbild als einem föderalen Gebilde. Insbesondere die aktuelle Sonderstellung der kurdischen Region erinnert stark an die ,asymmetrische“ Autonomie in Spanien, die sich u. a. in der privilegierten Stellung der „historischen Nationalitäten“ gegenüber den übrigen Regionen widerspiegelt. Im spanischen Fall hat sich die beschriebene Wirkungsweise bereits gezeigt. Dort hat das Autonomiesystem zur Herausbildung regionaler Identitäten selbst in Teilen des Landes beigetragen, wo diese zuvor kaum zu verzeichnen waren. Die fortwährenden Bestrebungen von Katalanen und Basken nach immer neuen Formen der Eigenständigkeit sollten als Warnung dienen, ein Autonomiemodell nach spanischem Vorbild als Optimum für den irakischen Staatsaufbau zu begreifen.

Vor diesem Hintergrund lässt sich auch die Kritik sunnitischer Vertreter nicht gänzlich von der Hand weisen. Diese befürchten ein Auseinanderbrechen des irakischen Staatsgebildes in einen kurdischen Norden, einen schiitischen Süden (als so genannte „Superregion" aller schiitischen Provinzen) und eine sunnitische Mitte, die keine nennenswerten Rohstoffressourcen vorzuweisen hätte. Eine Entscheidung darüber, in welche Richtung sich die Organisationsstruktur in den kommenden Jahren entwickeln wird, muss im Laufe der institutionellen Ausgestaltung des „Council of Union“ getroffen werden. Um ein Autonomiemodell $\mathrm{zu}$ verhindern und den zentrifugalen Tendenzen im Irak entgegenwirken zu können, sollte diese zweite Kammer mit starken Kompetenzen ausgestattet werden. Auf diese Weise könnte ein wirklich föderales Modell im Irak etabliert werden.

\section{Optimierungsmöglichkeiten des Transformationsprozesses}

Die beschriebenen Mängel können noch behoben werden, da der Verfassungsprozess nicht gänzlich abgeschlossen ist und das Verfassungsdesign nachverhandelt werden soll. Insbesondere stehen nun die zugesagten Verhandlungen über eine Verfassungsergänzung aus. Dabei wird es von zentraler Bedeutung sein, tatsächlich alle relevanten Gruppen, insbesondere die Sunniten, in die Verhandlungen einzubeziehen. Ein Konsens wird erneut 
äußerst schwierig herzustellen sein, weil die Mängel im ,föderalen“ System nicht auf Flüchtigkeitsfehlern beruhen, sondern den politischen Interessen der Kurden und Schiiten entsprechen. Eine Einigung mit den sunnitischen Vertretern ist daher nur schwer vorstellbar und würde weitgehende Zugeständnisse beider Seiten erfordern. Da aber - im Gegensatz zum Sommer 2005 - genügend zeitlicher Spielraum zur Verfügung steht, erscheint dies nicht gänzlich unmöglich. In diesem Verhandlungsprozess ist darüber hinaus ein weiteres Mal die internationale Gemeinschaft gefragt, mit Expertenwissen, öffentlichen Informationskampagnen und Diskussionsveranstaltungen auch die Bevölkerung in einen transparenten Entscheidungsprozess einzubeziehen.

Unabhängig von formalen Verfassungsänderungen ist ein Wandel der Verfassung, die bislang zahlreiche Aspekte ungeregelt lässt, durch deren legislative Konkretisierung zu erwarten. Dies lässt ebenfalls Raum für Korrekturen im Bereich des Verfassungsdesigns. Die erforderlichen Veränderungen wurden bereits angesprochen. Einige der Regelungen, wie das Regierungs- und das Wahlsystem, werden jedoch offenbar nicht als Problem wahrgenommen. Dies mag ein Trugschluss sein, da die Verfassungsverhandlungen hinter verschlossenen Türen stattfanden. Definitiv zählte aber die Form des Regierungs- und Wahlsystems nicht zu den umstrittenen Themen, die die öffentlichen Diskussionen dominierten. Dies ist erstaunlich, da es sich bei Fragen des Regierungs- und Wahlsystems immer auch um Machtfragen handelt. Andere Themen jedoch, wie die Rolle der Religion oder die Verteilung der Ressourcen, gelten als deutlich konfliktintensiver. Ihnen wird daher vermutlich ein weitaus höheres Maß an Aufmerksamkeit zukommen. Dies könnte sich allerdings als ein gravierender Fehler herausstellen.

Obgleich inhaltliche Probleme für den Augenblick schwerwiegende Konflikte hervorrufen und diese auch festigen können, sind es letztlich doch die institutionellen Regelungen, die den politischen Prozess langfristig steuern und damit strukturell Einfluss nehmen. Sie zu einem späteren Zeitpunkt zu verändern, würde ein hohes Maß an Konsens und politischem Willen erfordern. Daher erscheint der aktuelle Zeitpunkt, zu dem die Verfassung konkret ausgestaltet werden muss, als der geeignete, um langfristige Strukturreformen vorzunehmen, die den politischen Prozess dauerhaft stabilisieren können.

Letztlich entscheidet jedoch die Form ihrer Implementierung über die Wirkungsweise der verfassungsrechtlichen Regelungen. Damit ist nicht nur die tatsächliche Anwendung der Verfassungsnormen gemeint. Vielmehr entstehen durch politische Praxis und politische Übung in nahezu jedem demokratischen System der Welt Diskrepanzen zwischen Verfassungstext und Verfassungswirklichkeit. Dies ist bereits darin begründet, dass „,noch keine Verfassungsurkunde das Gebiet tatsächlicher verfassungsmäßiger Regierungsgewalt jemals erschöpft" hat (Hermens 1968: 156). Damit ist es durchaus vorstellbar, dass sich politische Konventionen herausbilden, die beispielsweise eine angemessene ethnische Repräsentation im Kabinett auf Dauer vorschreiben. Derartige Konventionen entfalten jedoch erst nach Jahren der politischen Praxis eine gewohnheitsrechtliche Wirkung und bieten zudem keine verfassungsrechtlichen Garantien. Dennoch könnte die Bildung der aktuellen „Regierung der nationalen Einheit“ ein Anfang sein. In jedem Fall ist sie als ein Erfolg zu werten, denn unter den gegebenen Umständen gleicht ihr Zustandekommen ei- 
nem nationalen Kraftakt. Insbesondere die zunehmende Gewalt zwischen Schiiten und Sunniten lässt die bislang noch zu beobachtende Kooperationsbereitschaft zwischen ihren führenden Akteuren erstaunlich erscheinen. Solange die Akteure zu einer solchen Kooperation bereit sind, bestehen weiterhin Chancen auf eine nachhaltige demokratische Transformation des Landes.

Damit fällt die Beantwortung der eingangs gestellten Frage, ob der Irak die historische Chance, die sich ihm bot, genutzt hat, zwiespältig aus. Weder der Entstehungsprozess noch das Verfassungsdesign konnten bislang den Anforderungen an einen erfolgreichen Verfassungsprozess gerecht werden. Da dieser Prozess jedoch nicht vollständig abgeschlossen ist, hat sich das Zeitfenster, in dem sich die Möglichkeiten einer verfassungsrechtlichen Konfliktregelung bieten, noch nicht wieder gänzlich geschlossen. Unter der Prämisse, dass der politische Prozess aufrechterhalten werden und ein offener Bürgerkrieg verhindert werden kann, besitzt der Irak immer noch die Chance, seine politischen Institutionen derart zu gestalten, dass sie seinen soziokulturellen Eigenschaften und politischen Herausforderungen gerecht werden.

\section{Literatur}

Austin, Alex/Fischer, Martina/Ropers, Norbert, 2004: Transforming Ethnopolitical Conflict. The Berghof Handbook, Wiesbaden.

Bächler, Günter, 2004: Conflict Transformation through State Reform, in: Austin/Fischer/ Ropers 2004, 273-294.

Benomar, Jamal, 2004: Constitution-Making After Conflict: Lessons for Iraq, in: Journal of Democracy, 15 (2), 81-95.

Brewer, Eric M./Moller, Sara B., 2006: Low Intensity Conflict and Nation-Building in Iraq: A Chronology, http://www.csis.org/component/option,com_csis_pubs/task,view/id,2990/, Download vom 25.05.06.

Brown, Nathan, 2005: The Final Draft of the Iraqi Constitution: Analysis and Commentary, http://www.carnegieendowment.org/publications/index.cfm?fa=view\&id=17423, Download vom 02.05.06.

Davis, Eric, 2005: Strategies for Promoting Democracy in Iraq, USIP Special Report No. 153, http://www.usip.org/pubs/specialreports/sr153.html, Download vom 15.05.06.

Dawisha, Adeed, 2005: The New Iraq. Democratic Institutions and Performance, in: Journal of Democracy, 16 (3), 35-49.

Dawisha, Adeed/Diamond, Larry, 2006: Iraq's Year of Voting Dangerously, in: Journal of Democracy, 17 (2), 89-103.

Eichhorst, Kristina, 2005: Ethnisch-separatistische Konflikte in Kanada, Spanien und Sri Lanka. Möglichkeiten und Grenzen institutioneller Konfliktregelungen, Frankfurt a. M. u. a. 
Eichhorst, Kristina/Sinjen, Svenja, 2005a: Der Irak nach den Wahlen, in: KAS, Analysen und Argumente 16/05.

Eichhorst, Kristina/Sinjen, Svenja, 2005b: Die irakische Übergangsverfassung: Fallstrick oder Chance für den Demokratisierungsprozess im Irak?, in: Björn Kilian/Christian Tobergte/Simon Wunder, Nach dem Dritten Golfkrieg: Sicherheitspolitische Analysen zu Verlauf und Folgen des Konflikts, Berlin, 55-69.

Eichhorst, Kristina/Sinjen, Svenja, 2006: Transformationsprozess im Irak - Option für eine Regierung der nationalen Einheit?, in: KAS-Auslandsinformationen, 03/06, 4-26.

Fish, Steven M., 2006: Stronger Legislatures, Stronger Democracies, in: Journal of Democracy, 17 (1), 5-20.

Hart, Vivien, 2003: Democratic Constitution Making, USIP Special Report, No. 107, http://www.usip.org/pubs/specialreports/sr107.pdf, Download vom 12.05.06.

Hermens, Ferdinand A., 1968: Verfassungslehre, Köln, Opladen.

Horowitz, Donald, 1985: Ethnic Groups in Conflict, Berkeley.

Horowitz, Donald, 1990: Making Moderation Pay: the Comparative Politics of Ethnic Conflict Management, in: Joseph V. Montville (Hrsg.), Conflict and Peacemaking in Multiethnic Societies, Lexington/Toronto, 451-475.

Independent Electoral Commission of Iraq, 2006: IECI Regulation 13-2005. Allcocation of Seats, http://www.ieciraq.org/final\%20cand/Regn13-2005AllocationofSeats_Engli sh-Dec6_\%5B1\%5D.pdf, Download vom 25.05.06.

International Crisis Group, 2005: Unmaking Iraq: A Constitutional Process Gone Awry, Middle East Briefing No. 19, http://www.icg.org, Download vom 17.04.06.

Lijphart, Arend, 1980: Democracy in Plural Societies. A Comparative Exploration, New Haven/London (2. Aufl.).

Lijphart, Arend, 1984: Democracies. Patterns of Majoritarian and Consensus Government in Twenty-One Countries, New Haven/London.

Lipset, Seymour M./Rokkan, Stein, 1967, Party Systems and Voter Alignments: Cross-National Perspectives, New York/London.

March, James G. / Olsen, Johan P., 1984: The New Institutionalism: Organizational Factors in Political Life, in: The American Political Science Review, Vol. 78, No. 3, (Sep. 1984), $734-749$.

Merkel, Wolfgang, 1999: Systemtransformation. Eine Einführung in die Theorie und Empirie der Transformationsforschung, Opladen.

Morrow, Jonathan, 2005: Iraq's Constitutional Process II: An Opportunity Lost, USIP Special Report No. 155, http://www.usip.org/pubs/specialreports/sr155.html\#secretariat, Download vom 02.04.06.

Popper, Karl, 1974: Das Elend des Historizismus, Tübingen (4. Aufl.).

Rayburn, Joel, 2006: The Last Exit From Iraq, in: Foreign Affairs, 85 (2), 29-40.

Reilly, Benjamin, 2001: Democracy in Divided Societies. Electoral Engineering for Conflict Management, Cambridge. 
Reilly, Benjamin, 2003: Political Engineering of Parties and Party Systems, http:// www.bt.undp.org/Democracy/Political\%20Engineering\%20of\%20Parties $\% 20$ and $\% 20$ Party\%20Systems.pdf, Download vom 15.05.06.

Sartori, Giovanni, 1976: Parties and Party Systems: A Framework for Analysis, Cambridge. Sartori, Giovanni, 1997: Comparative Constitutional Engineering. An Inquiry into Structures, Incentives and Outcomes, Houndmills u. a. (2. Aufl.).

Tipp, Charles, 2000: A History of Iraq, Cambridge.

USAID, 2006: Constitution Includes Voice of the People, http://www.usaid.gov/stories/ iraq/ss_iraq_constitution-people.html, Download vom 25.05.06.

Korrespondenzanschrift:

Dr. Kristina Eichhorst

Institut für Sicherheitspolitik

Christian-Albrechts-Universität zu Kiel

Olshausenstr. 40

24118 Kiel

E-Mail: keichhorst@isuk.org

\section{Die Verteidigung des Rechtstaates gegen den Terrorismus}

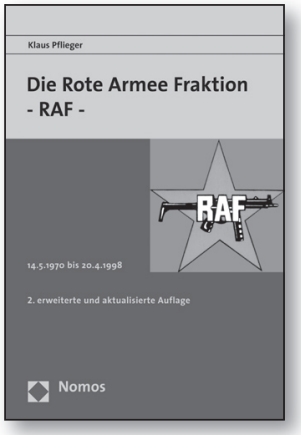

Die Rote Armee Fraktion - RAF -

14.5.1970 bis 20.4.1998

Von Klaus Pflieger, Generalstaatsanwalt, Stuttgart

2. erweiterte und aktualisierte Auflage 2006, $278 \mathrm{~S}$., brosch., 19,80 €, ISBN 978-3-8329-2207-8

"lch kenne kein anderes Buch, das eine so rasche und gleichzeitig präzise Orientierung über Namen und Geschehnisse der knapp 28jährigen Geschichte der RAF bietet."

Dr. Elke Koch, Zeitschrift für Württembergische Landesgeschichte 66/2007

Bitte bestellen Sie bei Ihrer Buchhandlung oder bei:

Nomos Verlagsgesellschaft | 76520 Baden-Baden | www.nomos.de 